\title{
Passive vehicle suspensions employing inerters with multiple performance requirements
}

\author{
Yinlong $\mathrm{Hu}^{\mathrm{a}}$, Michael Z. Q. Chen ${ }^{\mathrm{b}, *}$, Zhan $\mathrm{Shu}^{\mathrm{c}}$ \\ ${ }^{a}$ School of Automation, Nanjing University of Science and Technology, Nanjing, China \\ ${ }^{b}$ Department of Mechanical Engineering, The University of Hong Kong, Hong Kong \\ ${ }^{c}$ Faculty of Engineering and the Environment, University of Southampton, Southampton, U.K.
}

\begin{abstract}
This paper investigates passive vehicle suspensions with inerters by considering multiple performance requirements including ride comfort, suspension deflection and tyre grip, where suspension deflection performance is novelly considered which is formulated as a part of objective functions and a constraint separately. Six suspension configurations are analyzed and the analytical solutions for each performance measure are derived. The conditions for each configuration to be strictly better than the simpler ones are obtained by presenting the analytical solutions of each configuration based on those of the simpler ones. Then, two stages of comparisons are given to show the performance limitations of suspension deflection for passive suspensions with inerters. In the first stage, it is shown that although the configurations with inerters can improve the mixed performance of ride comfort and tyre grip, the suspension deflection performance is significantly decreased simultaneously. In the second stage, it is shown that for passive suspensions with inerters, suspension deflection is the more basic limitation for both ride comfort and tyre grip performance by doing comparisons among mixed ride comfort and suspension deflection optimization, mixed ride comfort and tyre grip optimization, and mixed suspension deflection and tyre grip optimization. Finally, the problem of mixed ride comfort and tyre grip performance optimization with equal suspension deflection is investigated. The limitations of suspension deflection for each configuration are further highlighted.
\end{abstract}

Keywords: Passive vehicle suspension; suspension deflection; inerter

\section{Introduction}

Vehicle suspension plays a central role in vehicle dynamics, contributing to improve the ride comfort and the vehicle stability [1]. Generally speaking, suspension systems can be classified into passive, semi-active and active suspensions. Passive suspensions are composed of only passive elements, such as springs, dampers and inerters [2]. Simplicity, high reliability, low cost and zero energy consumption are the advantages of passive suspensions. Semiactive suspensions are integrated with semi-active elements, such as the MR damper, the ER damper and the EH damper, of which the damping coefficient can be adjusted within a large

${ }^{*}$ Corresponding author. Email: mzqchen@hku.hk. 
actuation bandwidth by consuming only a small amount of energy [3, 4]. Active suspensions not only provide best performance but also demand most energy due to the force-generating actuators $[5,6]$.

The inerter is a recently proposed concept and device with the property that the applied force at the two terminals is proportional to the relative acceleration between them $[2,7]$. The inerter extends the class of mechanical realizations of complex impedances compared to the ones using only springs and dampers and has been applied to various mechanical systems, including vehicle suspensions $[8,9,10,11,12,13,14]$, motorcycle steering systems $[15,16]$, train suspensions $[17,18,19,20]$ and building vibration control [21, 22]. It has also rekindled interest in passive network synthesis [23, 24, 25, 26, 27].

The application of the inerter in passive suspension systems was first investigated in [8]. Improvements of about $10 \%$ or greater were announced in terms of ride comfort, type grip and dynamic load carrying performances for both quarter-car and full-car models. In [9], an ingenious approach to deriving analytical solutions for ride comfort and tyre grip performance measures in [8] in terms of six networks with fixed structures, which comprised one or two springs, one damper and possibly one inerter, was provided and it is demonstrated that the results in [8] were in fact global optima and the benefits of inerters were further highlighted. In [10], the nonlinearities of a ball-screw inerter were investigated and a nonlinear theoretical model was also obtained. An approach to optimizing all passive transfer functions (positive real admittances) with fixed order by the Linear Matrix Inequalities method was proposed in [11]. To keep the passive suspensions with inerter low complexity and low cost, the question what was the general class of vehicle suspension admittances which can be realized with only one damper, one inerter and arbitrary springs was answered in [12, 24]. Another consideration to realize higher-order admittance and also keep simplicity of vehicle suspension structure was to combine the mechanical and electrical networks together by using a novel mechatronic suspension strut which was composed of a ball-screw inerter and a Permanent Magnet Electric Machinery (PMEM) [13, 14].

It is well known that suspension deflection is one of the basic performance requirements for suspension systems, since large working space of suspensions will cause damages to vehicle components and generate more passenger discomfort [6]. Given the significant impact of suspension deflection on vehicle suspensions, the argument for equal suspension deflection comparisons has been suggested many years ago [28, 29, 30, 31]. However, for passive vehicle suspensions incorporating the inerter, suspension deflection has never been considered as a requirement $[8,9,10,11,12,13,14]$, and the influence of inerter on suspension deflection has not yet been clearly understood. Since the vehicle suspension design is a compromise among a number of factors, to fully investigate the performance of passive vehicle suspensions with inerters, multiple performance requirements including ride comfort, suspension deflection and tyre grip are considered for six simple suspension configurations in this paper. Suspension deflection performance is formulated as a part of the objective functions and a constraint separately. The performance limitations caused by suspension deflection requirement are demonstrated by carrying out different stages of comparisons. The issue of designing passive vehicle suspensions with equal suspension deflection is also investigated.

Note that the main differences between this paper and the existing works [8] and [9] are: 1. suspension deflection performance is novelly studied, which has never been investigated for passive vehicle suspensions with the inerter in the existing works, such as $[8,9,10,11,12,13$, 


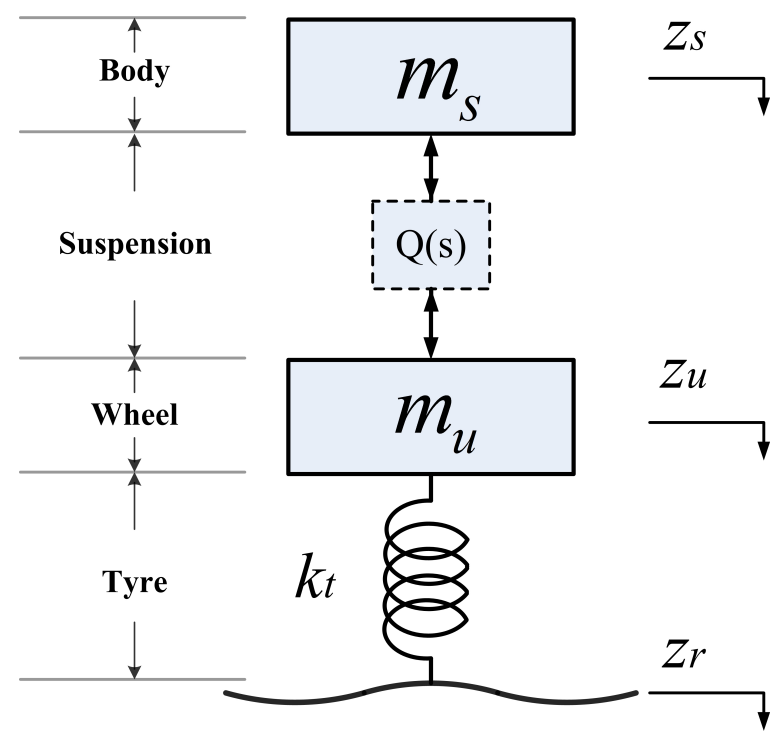

Figure 1: A quarter-car vehicle model.

14]. Two situations where suspension deflection is formulated as a part of objective functions and a hard constraint are investigated separately in this paper; 2 . The comparisons in this paper are carried out by considering multiple performance requirements simultaneously and the multi-objective optimization about ride comfort and tyre grip in [9] is further analyzed by inserting the requirement of equal suspension deflection performance; 3 . A different representation of the analytical solutions compared with [9] is given in this paper, where the conditions for each configuration to be strictly better than the simpler ones can directly be derived.

The rest of the paper is organized as follows. Section 2 introduces the relevant background on suspension structures and performance measures. Section 3 derives the analytical solutions for ride comfort, suspension deflection and tyre grip performances for each configuration, where a different representation of the solutions from that in [9] is given. Section 4 investigates the influences of suspension deflection by carrying out different stages of comparisons in mixed performance optimization. Section 5 investigates the passive vehicle suspensions employing inerters with equal suspension deflection requirement. Conclusions are drawn in Section 6.

\section{Vehicle model, suspension configurations, and performance measures}

The quarter-car model presented in Fig. 1 is the simplest model for suspension design. It consists of a sprung mass $m_{s}$, an unsprung mass $m_{u}$ and a tyre with spring stiffness $k_{t}$ [29]. Here, the suspension strut supplying an equal and opposite force on the sprung and unsprung masses is a passive mechanical admittance $Q(s)$ which is defined by the ratio of Laplace transformed force to relative velocity [8]. The suspension struts here are assumed to have negligible mass. The equations of motion in the Laplace domain are:

$$
\begin{aligned}
m_{s} s^{2} \hat{z}_{s} & =-s Q(s)\left(\hat{z}_{s}-\hat{z}_{u}\right), \\
m_{u} s^{2} \hat{z}_{u} & =s Q(s)\left(\hat{z}_{s}-\hat{z}_{u}\right)+k_{t}\left(\hat{z}_{r}-\hat{z}_{u}\right) .
\end{aligned}
$$




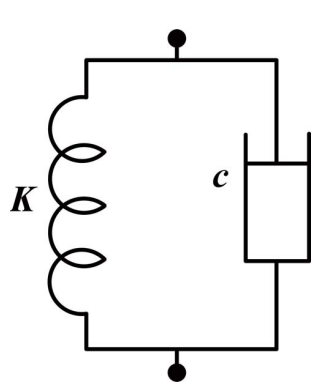

(a) $\mathrm{C} 1$

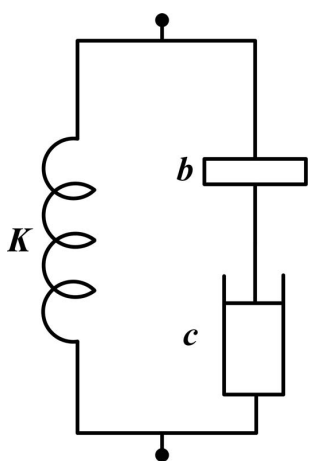

(d) $\mathrm{C} 4$

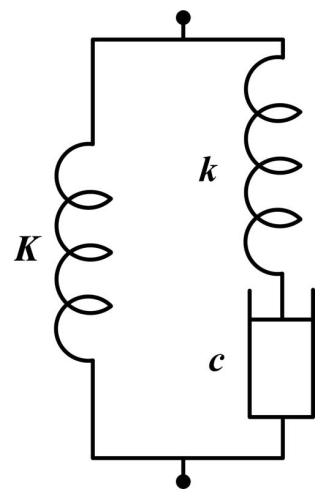

(b) $\mathrm{C} 2$

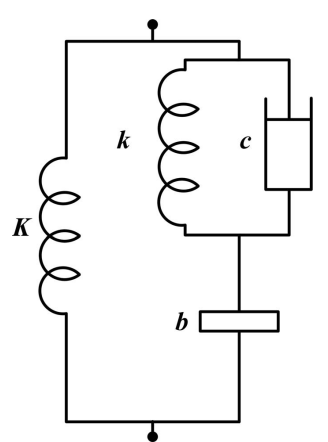

(e) C5

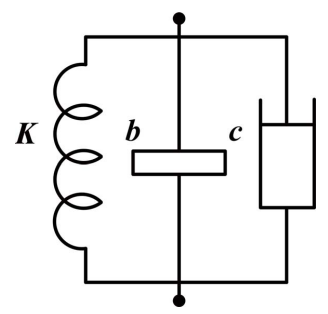

(c) $\mathrm{C} 3$

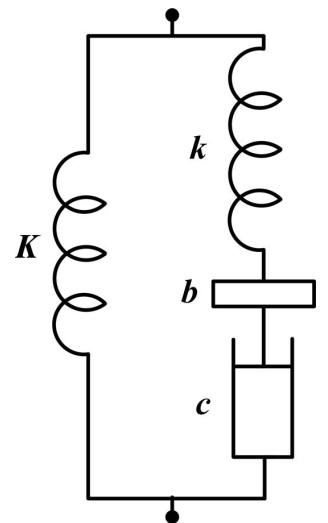

(f) $\mathrm{C} 6$

Figure 2: Six considered configurations. 
Table 1: $Q(s)$ for each configuration in Fig. 2.

\begin{tabular}{cll}
$Q_{1}(s)=\frac{K}{s}+c$ & $Q_{2}(s)=\frac{K}{s}+\frac{1}{\frac{s}{k}+\frac{1}{c}}$ & $Q_{3}(s)=\frac{K}{s}+b s+c$ \\
$Q_{4}(s)=\frac{K}{s}+\frac{1}{\frac{1}{b s}+\frac{1}{c}}$ & $Q_{5}(s)=\frac{K}{s}+\frac{1}{\frac{1}{s}+c}+\frac{1}{b s}$ & $Q_{6}(s)=\frac{K}{s}+\frac{1}{\frac{1}{c}+\frac{1}{b s}+\frac{s}{k}}$ \\
\hline
\end{tabular}

Fig. 2 shows the six configurations employed as suspension struts in this paper (corresponding $Q(s)$ given in Table 1), where $C 1, C 2$ are two struts without any inerter and each of the rest four contains an inerter. The performance measures used in this paper are discussed in detail in [32]. For ride comfort, we use the root-mean-square (rms) of body vertical acceleration in response to road disturbances, defined as $J_{1}$ as follows

$$
J_{1}=2 \pi(V \kappa)^{1 / 2}|| s T_{\hat{z}_{r} \rightarrow \hat{z}_{s}} \|_{2}
$$

where $V$ is the speed of the car, $\kappa$ is the road roughness parameter. $T_{\hat{z}_{r} \rightarrow \hat{z}_{s}}$ denotes the transfer function from the road disturbance $\hat{z}_{r}$ to the displacement of the sprung mass $\hat{z}_{s}$ and $\|\cdot\|_{2}$ is the standard $H_{2}$ norm. The rms suspension deflection parameter $J_{2}$ is defined as

$$
J_{2}=2 \pi(V \kappa)^{1 / 2}\left\|\frac{1}{s} T_{\hat{z}_{r} \rightarrow\left(\hat{z}_{s}-\hat{z}_{u}\right)}\right\|_{2} .
$$

The rms tyre grip parameter $J_{3}$ is defined as

$$
J_{3}=2 \pi(V \kappa)^{1 / 2}\left\|\frac{1}{s} T_{\hat{z}_{r} \rightarrow k_{t}\left(\hat{z}_{u}-\hat{z}_{r}\right)}\right\|_{2} .
$$

The parameters for the quarter car model and performance measures in this paper are (unless otherwise stated): $m_{s}=250 \mathrm{~kg}, k_{t}=150 \mathrm{kNm}^{-1}, m_{u}=35 \mathrm{~kg}, \kappa=5 \times 10^{-7} \mathrm{~m}^{3} \mathrm{cycle}^{-1}$, and $V=25 \mathrm{~ms}^{-1}$. The static stiffness $K$ is chosen from $10 \mathrm{kNm}^{-1}$ to $120 \mathrm{kNm}^{-1}$ which covers a range from softly sprung passenger cars through sports cars and heavy good vehicles up to racing cars [8].

\section{Individual performance analysis}

The analytical solutions for ride comfort and tyre grip performance measures have been derived in [9] by calculating $J_{i}, i=1,2,3$, as $J_{i}=2 \pi(V \kappa H)^{1 / 2}$. In this section, we derive the analytical solutions for suspension defection performance measure in the same manner (see [9] for details). However, an alternative representation for these solutions will be given in this paper, where the necessary and sufficient condition for each configuration to be strictly better than the simpler configuration can be derived. Such conditions can directly be used to justify whether it is necessary to upgrade a specific configuration to a more complex one in practice. 
The ride comfort performances are rewritten as

$$
\begin{aligned}
H_{C 1 J 1} & =d_{1} c+d_{2} c^{-1} \\
H_{C 2 J 1} & =H_{C 1 J 1}+\left(d_{3} k^{-1}+d_{4} k^{-2}\right) c \\
H_{C 3 J 1} & =H_{C 1 J 1}+\left(g_{3} b^{3}+g_{2} b^{2}+g_{1} b\right)\left(f_{1} b+f_{0}\right)^{-1} c^{-1}, \\
H_{C 4 J 1} & =H_{C 1 J 1}+\left(d_{5} b^{-1}+d_{6} b^{-2}\right) c \\
H_{C 5 J 1} & =H_{C 4 J 1}+\left(\left(d_{7}+d_{8} b^{-1}+d_{9} b^{-2}\right) k^{2}-\left(d_{5}+2 d_{6} b^{-1}\right) k\right) c^{-1}, \\
H_{C 6 J 1} & =H_{C 2 J 1}+\left(d_{5} b^{-1}+d_{6} b^{-2}-2 d_{2} b^{-1} k^{-1}\right) c, \\
& =H_{C 4 J 1}+\left(\left(d_{3}-2 d_{2} b^{-1}\right) k^{-1}+d_{4} k^{-2}\right) c,
\end{aligned}
$$

where parameters $d_{i}, i=1, \ldots, 9$ are given in Table 2 .

The following remarks can be obtained by analyzing the additional terms in (2)-(7):

Remark 1. (1) C2 provides no improvement for the ride comfort performance, as $d_{3}>0$ and $d_{4}>0$, which is consistent with the results in [8, 9].

(2) $C 3$ performs better than $C 1$ if and only if $b<b_{1}<\sqrt{4 K m_{s} m_{u} k_{t}^{-1}}$, where $b_{1}=$ $\left(\sqrt{g_{2}^{2}-4 g_{1} g_{3}}-g_{2}\right)\left(2 g_{3}\right)^{-1}$. Note that for a typical vehicle, the value of $b_{1}$ is relatively $s$ mall, such as $b_{1}=73.39 \mathrm{~kg}$ for the vehicle parameters employed in this paper with $K=60$ $\mathrm{kNm}^{-1}$. This explains the phenomenon revealed in [8, 9] that C3 requires small inertances for ride comfort performance. For example, the optimal inertance of $C 3$ in terms of ride comfort when $K=60 \mathrm{kNm}^{-1}$ is $31.27 \mathrm{~kg}$ as shown in [8] for the same vehicle.

(3) $C 4$ performs better than $C 1$ if and only if $b>-d_{6} / d_{5}=1 / 2\left(K / k_{t}+1 /\left(1+m_{u} / m_{s}\right)^{2}\right)\left(m_{s}+\right.$ $\left.m_{u}\right) \approx 1 / 2\left(m_{s}+m_{u}\right)$. Note that for a typical vehicle, the value of $-d_{6} / d_{5}$ is relatively large, such as $-d_{6} / d_{5}=166.60 \mathrm{~kg}$ for the vehicle parameters employed in this paper with $K=60$ $\mathrm{kNm}^{-1}$. This explains the phenomenon revealed in [8, 9] that $\mathrm{C} 4$ requires large inertances for the ride comfort performance. For example, the optimal inertance of $C 4$ in terms of ride comfort when $K=60 \mathrm{kNm}^{-1}$ is $333.30 \mathrm{~kg}$ as shown in [8] for the same vehicle.

(4) $C 5$ performs better than $C 4$ if and only if $b<-2 d_{6} / d_{5}$ and $k<\left(d_{5}+2 d_{6} b^{-1}\right)\left(d_{7}+\right.$ $\left.d_{8} b^{-1}+d_{9} b^{-2}\right)^{-1}$. Substituting the vehicle parameters employed in this paper and setting $K=60 \mathrm{kNm}^{-1}$, one obtains the conditions for C5 to perform better than $C 4$ is $b<333.3$ $\mathrm{kg}$ and $k<\left(9.12 \times 10^{4} b^{-1}-273.60\right)\left(0.0023-1.02 b^{-1}+268.63 b^{-2}\right)^{-1} \mathrm{Nm}^{-1}$. The optimal inertance of $C 5$ in terms of ride comfort when $K=60 \mathrm{kNm}^{-1}$ is about $200 \mathrm{~kg}$ as shown in [9], which satisfies the condition $b<333.3 \mathrm{~kg}$. For $b=200 \mathrm{~kg}$, the range of $k$ is $k<46.80$ $\mathrm{kNm}^{-1}$ which covers the optimal stiffness $\mathrm{k}$ (about $30 \mathrm{kNm}^{-1}$ as shown in [9]).

(5) $C 6$ performs better than $C 2$ if and only if $b>d_{6}\left(2 d_{2} k^{-1}-d_{5}\right)^{-1}$. For the vehicle parameters employed in this paper with $K=60 \mathrm{kNm}^{-1}$, the condition is $b>\left(360.04 k^{-1}+0.006\right)^{-1}$ $\mathrm{kg}$. C6 performs better than $C 4$ if and only if $b<K\left(m_{s}+m_{u}\right) k_{t}^{-1}$ and $k>d_{4}\left(2 d_{2} b^{-1}-d_{3}\right)^{-1}$, which explain why the relaxation spring in $C 6$ provides no improvement for the ride comfort performance as shown in [8, 9]: the optimal inertance $b$ in the series-connected arrangement always possesses a large value for ride comfort, and consequently, the condition $b<K\left(m_{s}+m_{u}\right) k_{t}^{-1}$ is not satisfied. For example, for the vehicle parameters employed in this paper with $K=60 \mathrm{kNm}^{-1}$, the condition for $C 6$ to perform better than $C 4$ is $b<114$ $\mathrm{kg}$ and $k>\left(3.8 b^{-1}-0.033\right)^{-1} k N m^{-1}$. As shown in [8], the optimal inertance of $C 4$ for the same vehicle when $K=60 \mathrm{kNm}^{-1}$ is $333.3 \mathrm{~kg}$, which does not satisfy the condition $b<114$ 
$\mathrm{kg}$, which means it is unnecessary to upgrade $C 4$ to $C 6$ by inserting a relaxation spring, or in other words, the relaxation spring is redundant for the ride comfort performance.

Proof. Since all the items in the remark are similarly derived by checking the additional terms in (2)-(7), for brevity, only the proofs of item (2) and item (3) are illustrated.

For item (2), since $f_{0}>0, f_{1}>0, g_{3}>0$ and $g_{1}<0$ as shown in Table 2 , then $H_{C 3 J 1}<H_{C 1 J 1}$ if and only if the additional term in (3) is negative, that is $g_{3} b^{2}+g_{2} b+g_{1}<0$, and then one obtains $b<b_{1}=\left(\sqrt{g_{2}^{2}-4 g_{1} g_{3}}-g_{2}\right)\left(2 g_{3}\right)^{-1}$ (here one assumes that $b>0$ and $c>0$, otherwise, the configuration reduces to a simpler one that is no longer the configuration $C 3)$.

Similarly, for item (3), since $d_{5}<0$ and $d_{6}>0$ as shown in Table 2 , then $H_{C 4 J 1}<H_{C 1 J 1}$ if and only if $d_{5} b^{-1}+d_{6} b^{-2}<0$, and then one obtains $b>-d_{6} / d_{5}$.

The suspension deflection performances are derived as

$$
\begin{aligned}
H_{C 1 J 2} & =e_{1} c^{-1}, \\
H_{C 2 J 2} & =H_{C 1 J 2}+e_{2} k^{-2} c, \\
H_{C 3 J 2} & =H_{C 1 J 2}, \\
H_{C 4 J 2} & =H_{C 1 J 2}+e_{3} b^{-2} c, \\
H_{C 5 J 2} & =H_{C 4 J 2}+\left(\left(e_{4} b^{-2}+e_{5} b^{-1}\right) k^{2}-2 e_{3} b^{-1} k\right) c^{-1}, \\
H_{C 6 J 2} & =H_{C 4 J 2}+\left(e_{2} k^{-2}-2 e_{1} k^{-1} b^{-1}\right) c, \\
& =H_{C 2 J 2}+\left(e_{3} b^{-2}-2 e_{1} k^{-1} b^{-1}\right) c,
\end{aligned}
$$

where parameters $e_{i}, i=1, \ldots, 5$ are given in Table 2 .

Similarly, for the suspension deflection performance measure, the following remarks can be obtained:

Remark 2. (1) For the individual performance $J_{2}$, the optimal values of each configuration are all zero by carefully choosing the coefficients of elements.

(2) $C 2, C 4$ and $C 6$ provide no improvement for suspension deflection compared with $C 1$, and $C 3$ achieves equal suspension deflection with $C 1$.

(3) $C 5$ performs better than $C 4$ if and only if $k<2 e_{3}\left(e_{4} b^{-1}+e_{5}\right)^{-1}$. For the vehicle parameters employed in this paper with $K=60 \mathrm{kNm}^{-1}$, the condition is $k<\left(2.95 b^{-1}+5.48 \times 10^{-3}\right)^{-1}$ $k N m^{-1}$. C5 performs better than $C 1$ if and only if $k<2 e_{3}\left(e_{4} b^{-1}+e_{5}\right)^{-1}$ and $c<2 k b-$ $\left(e_{4} / e_{3}+e_{5} / e_{3} b\right) k^{2}$. For the vehicle parameters employed in this paper with $K=60 \mathrm{kNm}^{-1}$, the condition is $k<\left(2.95 b^{-1}+5.48 \times 10^{-3}\right)^{-1} k N m^{-1}$ and $c<2 k b-\left(0.0059+1.10 \times 10^{-5} b\right) k^{2}$ $\mathrm{Nsm}^{-1}$.

(4) C6 performs better than $C 4$ if and only if $k>e_{2} b\left(2 e_{1}\right)^{-1}$. For the vehicle parameters employed in this paper with $K=60 \mathrm{kNm}^{-1}$, the condition is $k>263.16 \mathrm{~b} \mathrm{Nm^{-1 }}$. C6 performs better than $C 2$ if and only if $b>e_{3} k\left(2 e_{1}\right)^{-1}$. For the vehicle parameters employed in this paper with $K=60 \mathrm{kNm}^{-1}$, the condition is $b>0.0028 \mathrm{~kg}$.

Proof. The proof is similar to Remark 1, hence omitted. 
The tyre grip performances are rewritten as

$$
\begin{aligned}
H_{C 1 J 3} & =a_{1} c+a_{2} c^{-1} \\
H_{C 2 J 3} & =H_{C 1 J 3}+\left(a_{3} k^{-1}+a_{4} k^{-2}\right) c, \\
H_{C 3 J 3} & =H_{C 1 J 3}+\left(a_{5} b^{2}-a_{3} b\right) c^{-1}, \\
H_{C 4 J 3} & =H_{C 1 J 3}+\left(a_{6} b^{-1}+a_{7} b^{-2}\right) c, \\
H_{C 5 J 3} & =H_{C 4 J 3}+\left(\left(a_{8}+a_{9} b^{-1}+a_{10} b^{-2}\right) k^{2}-\left(a_{6}+2 a_{7} b^{-1}\right) k\right) c^{-1}, \\
H_{C 6 J 3} & =H_{C 2 J 3}+\left(a_{6} b^{-1}+a_{7} b^{-2}-2 a_{2} b^{-1} k^{-1}\right) c, \\
& =H_{C 4 J 3}+\left(\left(a_{3}-2 a_{2} b^{-1}\right) k^{-1}+a_{4} k^{-2}\right) c,
\end{aligned}
$$

where parameters $a_{i}, i=1, \ldots, 10$ are given in Table 2 .

For tyre grip performance measure, the following remarks can be obtained:

Remark 3. (1) $C 2$ performs better than $C 1$ if and only if $K<m_{s}\left(m_{s}+2 m_{u}\right) k_{t} /\left(2\left(m_{s}+m_{u}\right)^{2}\right)$ and $k>-a_{4} / a_{3}$. For the vehicle parameters employed in this paper, the first inequality is $K<73.87 \mathrm{kNm}^{-1}$, and if setting $K=60 \mathrm{kNm}^{-1}$, the second inequality is $k>434.38 \mathrm{kNm}^{-1}$. One sees that $C 2$ improves the tyre grip performance over $C 1$ for soft suspensions and the required relaxation spring stiffness is quite large, which is consistent with the results in [8, 9] (the optimal $k$ of $C 2$ for tyre grip when $K=60 \mathrm{kNm}^{-1}$ is about $700 \mathrm{kNm}^{-1}$, as shown in [9] for the same vehicle).

(2) $C 3$ performs better than $C 1$ if and only if $K>m_{s}\left(m_{s}+2 m_{u}\right) k_{t} /\left(2\left(m_{s}+m_{u}\right)^{2}\right)$ and $b<a_{3} / a_{5}$. For the vehicle parameters employed in this paper, the first inequality is $K>73.87$ $\mathrm{kNm}^{-1}$, and if setting $K=80 \mathrm{kNm}^{-1}$, the second inequality is $b<23.30 \mathrm{~kg}$. One sees that C3 improves the tyre grip performance over $C 1$ for stiff suspensions and the required inertance is quite small, which is consistent with the results in [8, 9] (the optimal b of C3 for tyre grip when $K=80 \mathrm{kNm}^{-1}$ is about $20 \mathrm{~kg}$, as shown in [9] for the same vehicle).

(3) $C 4$ performs better than $C 1$ if and only if $K>m_{s} m_{u} k_{t} /\left(m_{s}+m_{u}\right)^{2}$ and $b>-a_{7} / a_{6}$. For the vehicle parameters employed in this paper, the first inequality is $K>16.16 \mathrm{kNm}^{-1}$, and if setting $K=60 \mathrm{kNm}^{-1}$, the second inequality is $b>191.71 \mathrm{~kg}$. One sees that $C 4$ improves the tyre grip performance over $C 1$ for stiff suspensions and the required inertance is quite large (the optimal b of C4 for tyre grip when $K=60 \mathrm{kNm}^{-1}$ is about $400 \mathrm{~kg}$, as shown in [9] for the same vehicle). Note that compared with $C 3$, the range of the static stiffness for $C 4$ to be better than $C 1$ is larger than that for $C 3$.

(4) C5 performs better than $C 4$ if and only if $k<\left(a_{6}+2 a_{7} b^{-1}\right)\left(a_{8}+a_{9} b^{-1}+a_{10} b^{-2}\right)^{-1}$ and $b<-2 a_{7} / a_{6}$. For the vehicle parameters employed in this paper with $K=60 \mathrm{kNm}^{-1}$, the conditions are $b<383.42 \mathrm{~kg}$ and $k<\left(6.23 \times 10^{7} b^{-1}-1.65 \times 10^{5}\right)\left(1.82 \times 10^{5} b^{-2}-715 b^{-1}+\right.$ $1.85)^{-1} \mathrm{Nm}^{-1}$. As shown in [9] for the same vehicle, the optimal inertance $b$ of $C 5$ for tyre grip when $K=60 \mathrm{kNm}^{-1}$ is about $200 \mathrm{~kg}$, and for $b=200 \mathrm{~kg}$, the range of $k$ is $k<52.63$ $\mathrm{kNm}^{-1}$ (the optimal $k$ is about $40 \mathrm{kNm}^{-1}$, as shown in [9] for the same vehicle).

(5) C6 performs better than $C 2$ if and only if $b>a_{7}\left(2 a_{2} k^{-1}-a_{6}\right)^{-1}$, and for the vehicle parameters employed in this paper with $K=60 \mathrm{kNm}^{-1}$, the condition is $b>\left(450.58 \mathrm{k}^{-1}+\right.$ $0.0052)^{-1}$. C6 performs better than $C 4$ if and only if $k>a_{4}\left(2 a_{2} b^{-1}-a_{3}\right)^{-1}$, and for the vehicle parameters employed in this paper with $K=60 \mathrm{kNm}^{-1}$, the condition is $k>\left(1.19 b^{-1}+2.30 \times\right.$ $\left.10^{-3}\right)^{-1} \mathrm{kNm}^{-1}$. The optimal inertance $b$ and stiffness $k$ when $K=60 \mathrm{kNm}^{-1}$ are about 300 $\mathrm{kg}$ and $300 \mathrm{kNm}^{-1}$, respectively, as shown in [9] for the same vehicle. For $k=300 \mathrm{kNm}^{-1}$, 
Table 2: Summary of parameters.

\begin{tabular}{|c|c|}
\hline $\begin{aligned} d_{1}= & \frac{k_{t}}{2 m_{s}^{2}} \quad d_{2}=\frac{\left(m_{s}+m_{u}\right) K^{2}}{2 m_{s}^{2}} \\
d_{3}= & \frac{k_{t} K}{m_{s}^{2}} \quad d_{4}=\frac{k_{t} K^{2}}{2 m_{s}^{2}} \\
e_{4}= & \frac{\left(m_{s}+m_{u}\right)^{3} K^{2}+2\left(m_{s}+m_{u}\right) k_{t} m_{s}^{2} K+k_{t}^{2} m_{s}^{3}}{2 K^{2} k_{t}^{2}} \\
d_{6}= & \frac{\left(m_{s}+m_{u}\right)^{2} K^{2}+k_{t} m_{s}^{2} K}{2 m_{s}^{2} k_{t}} \quad d_{7}=\frac{m_{s}+m_{u}}{2 m_{s}^{2}} \\
d_{8}= & -\frac{2\left(m_{s}+m_{u}\right)^{2} K+m_{s}^{2} k_{t}}{2 m_{2}^{2} k_{t}} \\
d_{9}= & \frac{\left(m_{s}+m_{u}\right)^{3} K^{2}+2 m_{s}^{2}\left(m_{s}+m_{u}\right) k_{t} K+m_{s}^{3} k_{t}^{2}}{2\left(m_{s} k_{t}\right)^{2}} \\
g_{2}= & -2 k_{t}\left(m_{s}+m_{u}\right) K+m_{s} k_{t}^{2} \\
a_{2}= & \frac{\left(m_{s}+m_{u}\right)^{3} K^{2}}{2 m_{s}^{2}}-\frac{m_{u}\left(m_{s}+m_{u}\right) k_{t} K}{m_{s}}+\frac{m_{u} k_{t}^{2}}{2} \\
a_{4}= & \frac{\left(m_{s}+m_{u}\right)^{2} k_{t} K^{2}-m_{s}\left(m_{s}+2 m_{u}\right) k_{t}^{2} K+m_{s}^{2} k_{t}^{3}}{2 m_{s}^{2}} \\
a_{9}= & \frac{-2\left(m_{s}+m_{u}\right)^{4} K+m_{s}\left(m_{s}+m_{u}\right)^{2}\left(2 m_{u}-m_{s}\right) k_{t}}{2 m_{s}^{2} k_{t}} \\
a_{7}= & \frac{\left(m_{s}+m_{u}\right)^{4} K^{2}+\left(m_{s}+m_{u}\right)^{2}\left(m_{s}-2 m_{u}\right) m_{s} k_{t} K+(1}{2 m_{s}^{2} k_{t}} \\
a_{10}= & \frac{m_{s}+m_{u}}{2 m_{s}^{2} k_{t}^{2}}\left(\left(m_{s}+m_{u}\right)^{4} K^{2}+2 m_{s}\left(m_{s}-1\right.\right. \\
& \left.\quad+\left(m_{u}^{2}-m_{s} m_{u}+m_{s}^{2}\right)\left(m_{s} k_{t}\right)^{2}\right)\end{aligned}$ & $\begin{array}{l}e_{1}=\frac{m_{s}+m_{u}}{2} \quad e_{2}=\frac{k_{t}}{2} \\
e_{3}=\frac{\left(m_{s}+m_{u}\right)^{2} K+m_{s}^{2} k_{t}}{2 k_{t} K} \\
d_{5}=-\frac{\left(m_{s}+m_{u}\right) K}{m_{s}^{2}} \quad e_{5}=\frac{m_{s}^{2}}{2 K^{2}} \\
f_{0}=2 m_{s}^{3} m_{u} \quad f_{1}=2\left(m_{s}+m_{u}\right) m_{s}^{2} \\
g_{0}=m_{s} m_{u}\left(m_{s}+m_{u}\right) K^{2} \\
g_{1}=-2 m_{s} m_{u} k_{t} K \\
g_{3}=k_{t}^{2} \quad a_{1}=\frac{\left(m_{s}+m_{u}\right)^{2} k_{t}}{2 m_{s}^{2}} \\
a_{3}=\frac{2\left(m_{s}+m_{u}\right)^{2} k_{t} K-m_{s}\left(m_{s}+2 m_{u}\right) k_{t}^{2}}{2 m_{s}^{2}} \\
a_{6}=\frac{-\left(m_{s}+m_{u}\right)^{3} K+m_{s} m_{u}\left(m_{s}+m_{u}\right) k_{t}}{m_{s}^{2}} \\
a_{5}=\frac{\left(m_{s}+m_{u}\right) k_{t}^{2}}{2 m_{s}^{2}} a_{8}=\frac{\left(m_{s}+m_{u}\right)^{3}}{2 m_{s}^{2}} \\
\left.n_{s} m_{u} k_{t}\right)^{2} \\
\left.-m_{u}\right)^{2}\left(m_{s}-m_{u}\right) k_{t} K\end{array}$ \\
\hline
\end{tabular}

the range of $b$ is $b>148.85 \mathrm{~kg}$, and for $b=300 \mathrm{~kg}$, the range of $k$ is $k>159.15 \mathrm{kNm}^{-1}$. It is clear that the optimal inertance and spring stiffness satisfy the conditions derived in this paper.

Proof. The proof is similar to Remark 1, hence omitted.

\section{Suspension deflection performance in mixed performance optimization}

The mixed $J_{1}$ and $J_{3}$ performance optimization has been done in [9] by defining a mixed performance measure of $J_{1}$ and $J_{3}$ as follows:

$$
H_{C i ; 1,3}=(1-\alpha) m_{s}^{2} H_{C_{i} J_{1}}+\alpha H_{C_{i} J_{3}}, \alpha \in[0,1]
$$

In this section, mixed $J_{1}$ and $J_{2}$ performance measure $H_{C i ; 1,2}$, and mixed $J_{2}$ and $J_{3}$ performance measure $H_{C i ; 2,3}$ are defined in a similar manner as

$$
\begin{aligned}
& H_{C i ; 1,2}=(1-\alpha) H_{C i J 1}+\alpha m_{s}^{2} m_{u} H_{C i J 2}, \\
& H_{C i ; 2,3}=(1-\alpha) m_{s}^{4} H_{C i J 2}+\alpha H_{C i J 3},
\end{aligned}
$$

where $\alpha \in[0,1], H_{C_{i} J_{1}}, H_{C_{i} J_{2}}$, and $H_{C_{i} J_{3}}$ are given in the previous section, and $m_{s}^{2} m_{u}, m_{s}^{4}$ are inserted to approximately normalize the measures.

Note that the mixed performance measures defined in (22), (23) and (24) for each configuration can be represented in a similar form as in Section 3, where by analyzing the additional terms, the explicit conditions for each configuration to be better than the simpler ones can similarly be obtained. For brevity, they are not shown in this paper. The main focus of this section is to investigate the influence of the inerter on suspension deflection performance 


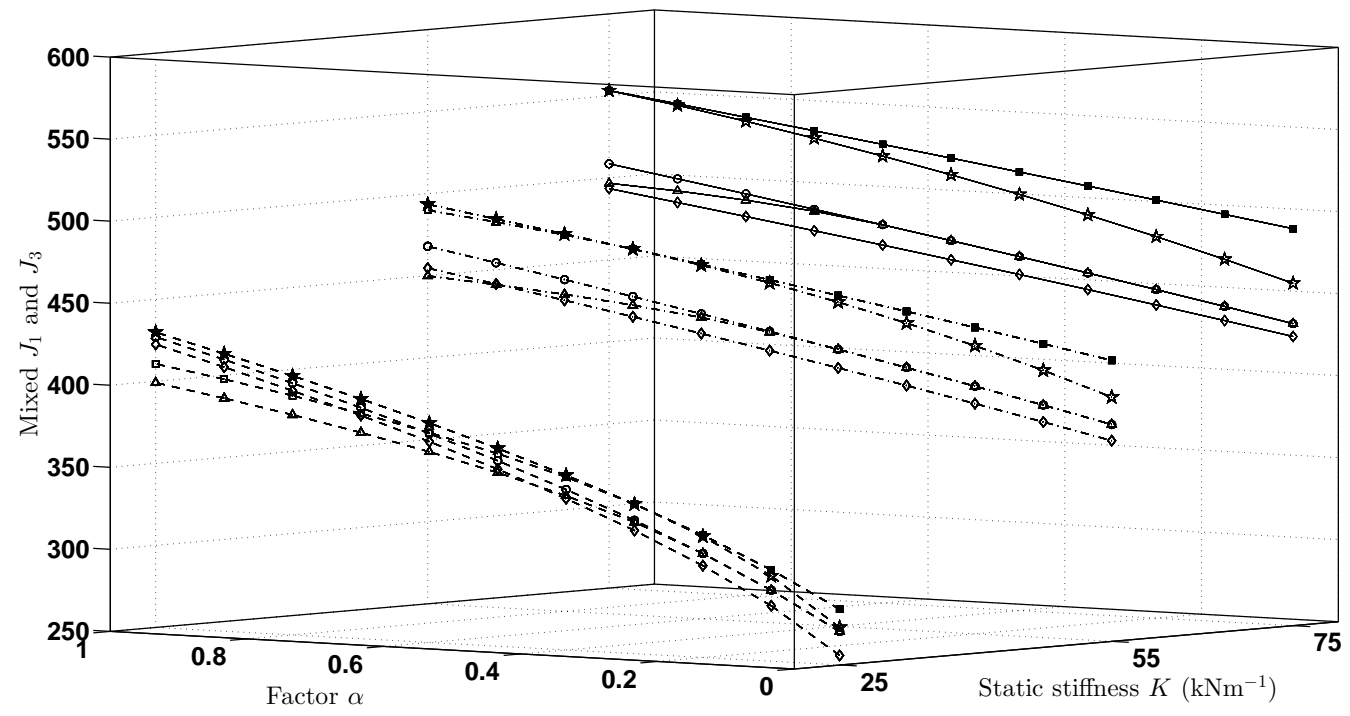

Figure 3: Mixed $J_{1}$ and $J_{3}$ performance. Solid, dot-dash, and dash lines denote $K=75 \mathrm{kNm}^{-1}, K=55$ $\mathrm{kNm}^{-1}$, and $K=25 \mathrm{kNm}^{-1}$, respectively. Solid circles denote $C 1$; squares denote $C 2$; stars denote $C 3$; hollow circles denote $C 4$; diamonds denote $C 5$; triangles denote $C 6$.

and to demonstrate the necessity of considering suspension deflection in vehicle suspension design. Hence, in what follows, two stages of comparisons will be given.

At the first stage of comparison, the suspension deflection performance measures $J_{2}$ for each configuration by using the parameters in the mixed $J_{1}$ and $J_{3}$ optimization are compared. Fig. 3 shows the optimization results of mixed $J_{1}$ and $J_{3}$ performance with respect to different $\alpha$ and different static stiffness $K$, where we see that the configurations with inerters can greatly improve the mixed $J_{1}$ and $J_{3}$ performance compared with $C 1$ and $C 2$. However, as shown in Fig. 4 , the $J_{2}$ performance is significantly degraded, which means that the mixed $J_{1}$ and $J_{3}$ performance is improved by sacrificing the suspension deflection performance.

At the second stage of comparison, the $J_{1}$ performance in mixed $J_{1}$ and $J_{3}$ optimization will be compared with the $J_{1}$ performance in mixed $J_{1}$ and $J_{2}$ optimization to show which is the more basic limitation for $J_{1}$ performance in terms of $J_{2}$ and $J_{3}$. Besides, the $J_{3}$ in mixed $J_{1}$ and $J_{3}$ optimization will be compared with $J_{2}$ in mixed $J_{2}$ and $J_{3}$ optimization to show which is the more basic limitation for $J_{3}$ performance in terms of $J_{1}$ and $J_{2}$. For the mixed $J_{1}$ and $J_{2}$ optimization and mixed $J_{2}$ and $J_{3}$ optimization, the optimal solutions can be derived by doing some algebraical calculations, where the analytical results are given in Appendices A and B.

Since similar results are obtained for these configurations, for simplicity, only the results of $C 5$ are shown in Fig. 5 and Fig. 6. In Fig. 5 , it is shown that the $J_{1}$ performance in mixed $J_{1}$ and $J_{2}$ optimization is always (significantly) larger than that in mixed $J_{1}$ and $J_{3}$ optimization, which means that suspension deflection is a more basic limitation than tyre grip for ride comfort performance. Similarly, as shown in Fig. 6, suspension deflection is also a more basic limitation than ride comfort for tyre grip performance. 


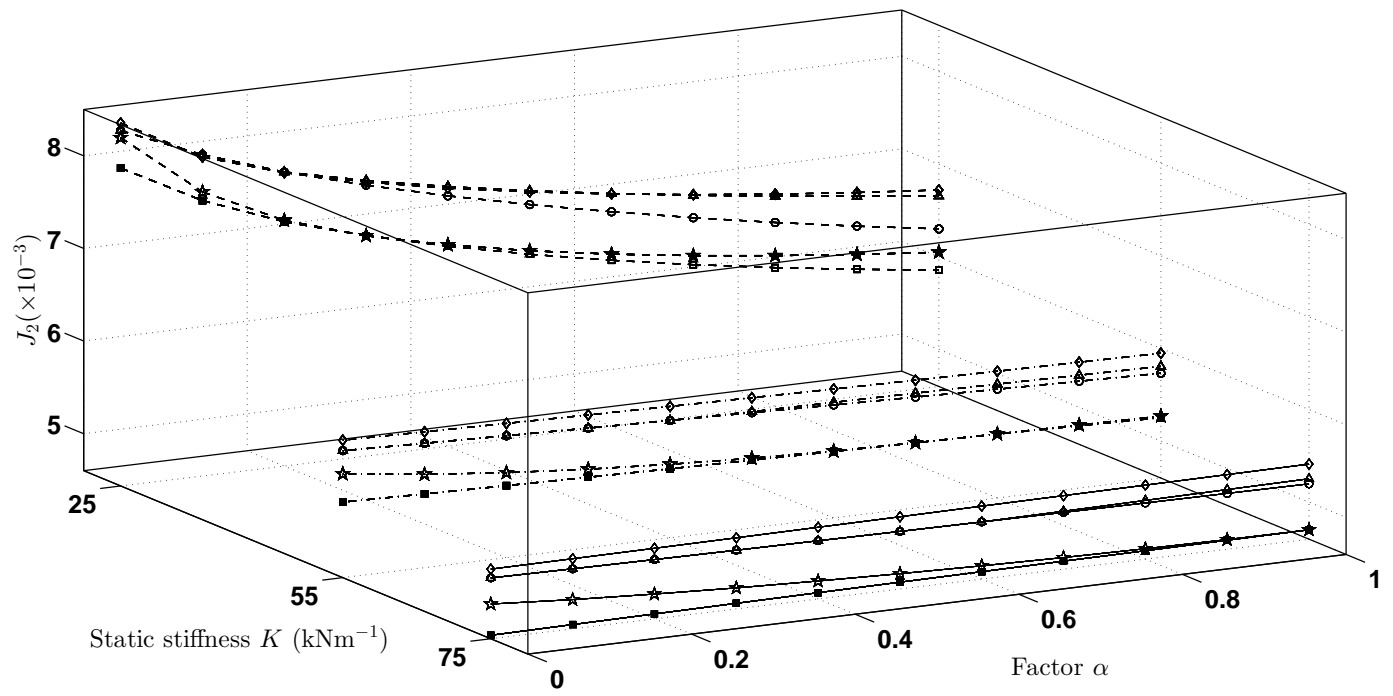

Figure 4: $J_{2}$ performance in mixed $J_{1}$ and $J_{3}$ optimization. Solid, dot-dash, and dash lines denote $K=75$ $\mathrm{kNm}^{-1}, K=55 \mathrm{kNm}^{-1}$, and $K=25 \mathrm{kNm}^{-1}$, respectively. Solid circles denote $C 1$; squares denote $C 2$; stars denote $C 3$; hollow circles denote $C 4$; diamonds denote $C 5$; triangles denote $C 6$.

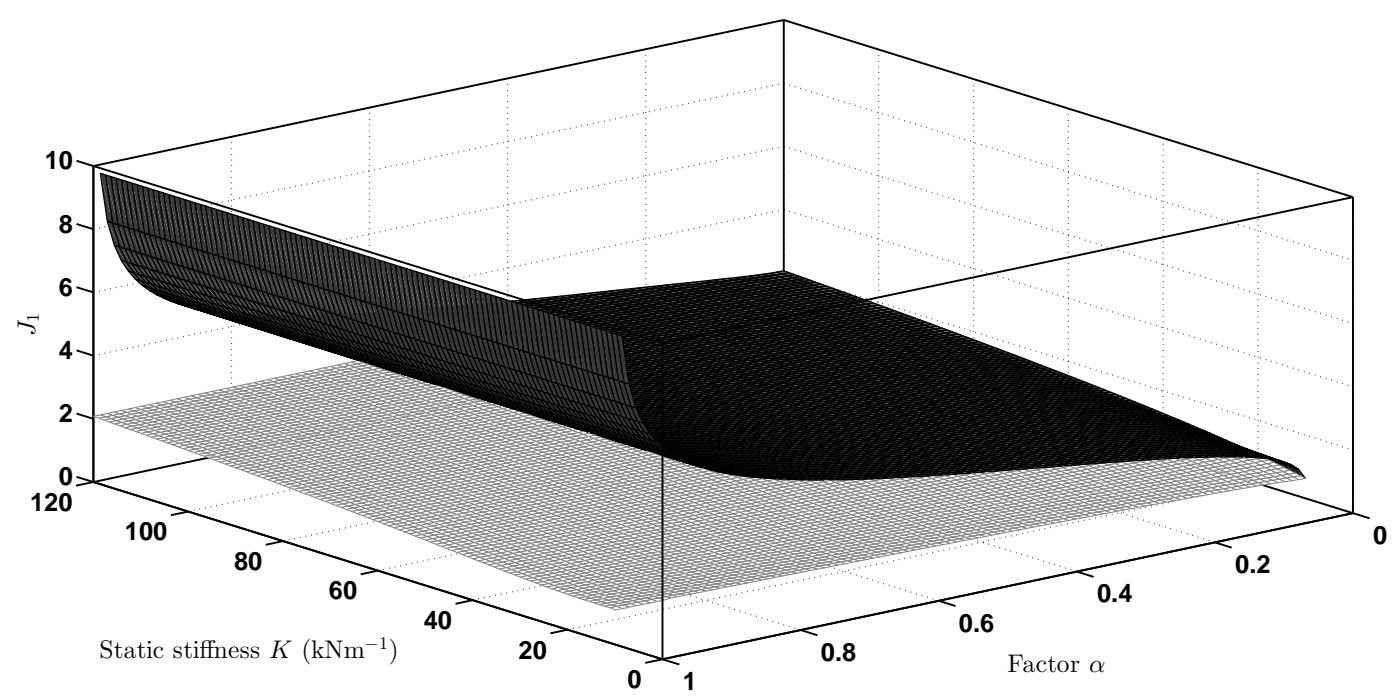

Figure 5: Comparison of $J_{1}$ in mixed $J_{1}, J_{2}$ optimization and mixed $J_{1}, J_{3}$ optimization for $C 5$. Light color denotes the $J_{1}$ in mixed $J_{1}$ and $J_{3}$ optimization; dark color denotes the $J_{1}$ in mixed $J_{1}$ and $J_{2}$ optimization. 


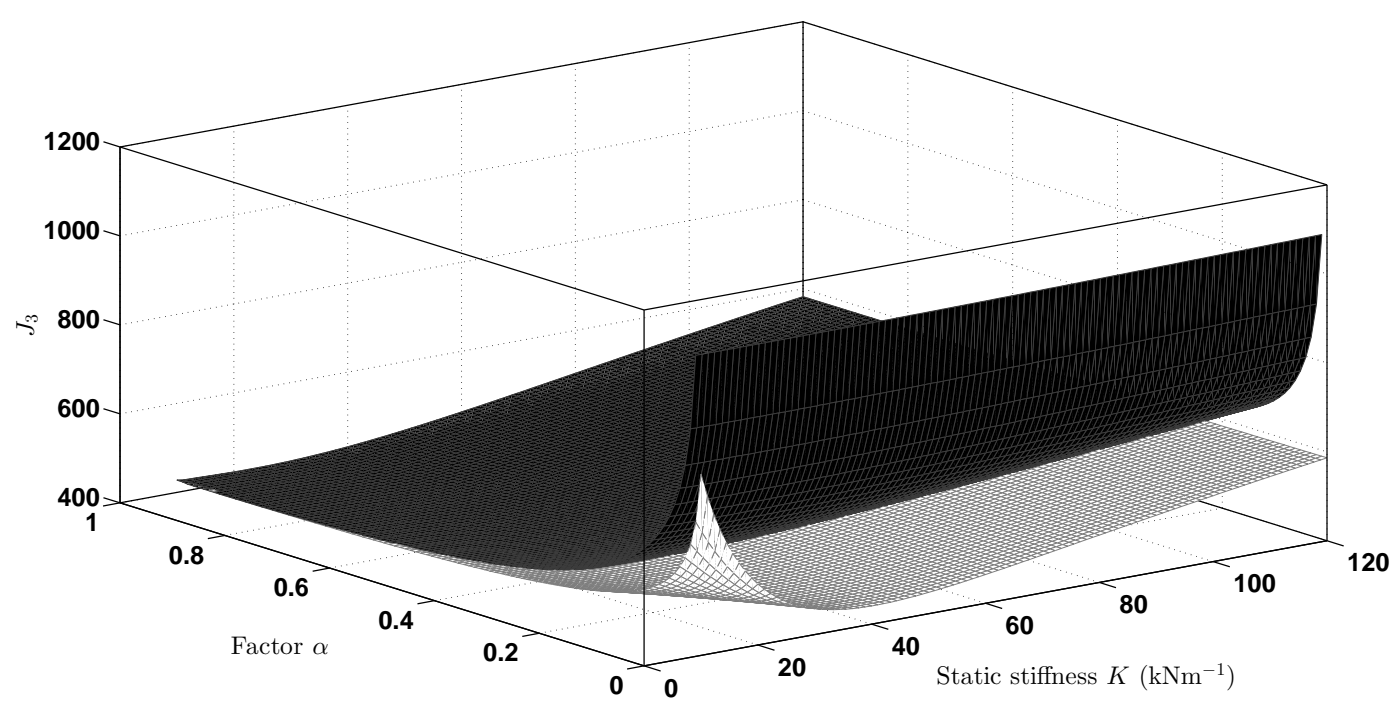

Figure 6: Comparison of $J_{3}$ in mixed $J_{1}, J_{3}$ optimization and mixed $J_{2}, J_{3}$ optimization for $C 5$. Light color denotes the $J_{3}$ in mixed $J_{1}$ and $J_{3}$ optimization; dark color denotes the $J_{3}$ in mixed $J_{2}$ and $J_{3}$ optimization.

\section{Vehicle suspensions with equal suspension deflection performance}

From Section 4, one sees that suspension deflection is a basic limitation for both ride comfort and tyre grip performance and the improvements in the mixed ride comfort and tyre grip optimization are actually obtained by reducing the suspension deflection performance simultaneously. Hence, in this section, an optimization problem will be proposed to realize the equal suspension deflection design by using the analytical solutions derived in this paper.

The optimization problems are formulated as

$$
\begin{array}{rc}
\min _{b, c, k} J_{C i ; 1,3} & \\
J_{C i ; 1,3} & =2 \pi\left(V \kappa\left((1-\alpha) m_{s}^{2} H_{C i J 1}+\alpha H_{C i J 3}\right)\right)^{1 / 2}, \\
\text { subject to } & J_{2 ; C i} \leq \gamma, i=1, \ldots, 6
\end{array}
$$

where $\gamma$ is the permitted largest rms value of $J_{2}$ and $J_{2 ; C i}=2 \pi\left(V \kappa H_{C i J 2}\right)^{1 / 2}, H_{C i J 1}, H_{C i J 2}$ and $H_{C i J 3}$ are given in (1)-(21).

Note that the problems for $C 1$ and $C 3$ are easy due to the simple representations of $J_{2}$ as shown in Equations (8) and (10), where the constraint $J_{2 ; C i} \leq \gamma$ can be transformed into $c \geq$ $\left(4 \pi^{2} V \kappa e_{1}\right) / \gamma^{2}$ and the problems become unconstrained ones. For the other configurations, the constrained nonlinear optimization function fmincon in Matlab is used with various starting points to guarantee the global optima. Since the performances for soft and stiff suspensions are different, we illustrate the static stiffness with $K=20 \mathrm{kNm}^{-1}$ and $K=80 \mathrm{kNm}^{-1}$ separately.

Fig. 7 and Fig. 8 show the results with $\alpha=0.5$, where one sees that the mixed performance measures of $J_{1}$ and $J_{3}$ are significantly reduced by restricting the $J_{2}$ performance at the same level. For example, $3.72 \%$ improvements can be achieved for $C 6$ if there is no limitation of suspension deflection $(\gamma>=0.008)$ when $K=20 \mathrm{kNm}^{-1}$, while such improvements will be reduced to $1.67 \%$ if the constraint $J_{2}<=0.004$ is imposed, as shown in Fig. 7. Observing Fig. 8(b), one can see that although $C 6$ reduces to $C 4$ if suspension deflection is not a 
limitation $(\gamma>=0.005)$, for $\gamma<0.005, C 6$ always performs better than $C 4$, which indicates that with the equal suspension deflection requirement, the optimal behaviors of some configurations may be different from the cases without such a requirement. Hence, it is essential to take suspension deflection into account in vehicle suspension design. The comparisons of the Pareto optimal solutions for $J_{1}$ and $J_{3}$ with and without the equal suspension deflection requirement are depicted in Fig. 9 and Fig. 10, where the performance degradations of $J_{1}$ and $J_{3}$ are clearly shown for each configuration.

\section{Conclusion}

This paper has investigated the problem of passive vehicle suspension design with inerters by considering multiple performance requirements including ride comfort, suspension deflection and tyre grip, where the suspension deflection performance was formulated as a part of the objective functions and a constraint separately. The analytical solutions for six suspension configurations have been derived and an alternative representation of these solutions has been given, where the explicit conditions for each configuration to be strictly better than the simpler ones can directly be derived. To investigate the influence of suspension deflection in passive vehicle suspensions with inerters, two stages of comparisons have been carried out. At the first stage, the suspension deflection performance measures for the configurations under consideration with the parameters obtained in the mixed ride comfort and tyre grip optimization were compared. The result showed that although the configurations with inerters can improve the mixed performance measure of ride comfort and tyre grip, the suspension deflection performance was significantly reduced simultaneously. At the second stage, for all the configurations, the ride comfort performances in mixed ride comfort and suspension deflection optimization and in mixed ride comfort and tyre grip optimization were compared. Meanwhile, the tyre grip performances in mixed ride comfort and tyre grip optimization and mixed suspension deflection and tyre grip optimization were also compared. It was shown in this stage that for passive suspensions with inerters, suspension deflection is the more basic limitation for both ride comfort and tyre grip performance. Finally, the problem of equal suspension deflection comparison was considered, where the mixed ride comfort and tyre grip performance optimization was integrated with a hard constraint on suspension deflection. The performance limitations of suspension deflection for each configuration were further highlighted.

\section{Acknowledgments}

This work is supported by Hong Kong University Committee on Research and Conference Grants under Grant 201111159110, National Natural Science Foundation of China under Grant 61004093, and by National Key Basic Research Scheme of China("973" Scheme) under Grant 2012CB720202.

\section{Appendix A. Analytical solutions of mixed $J_{1}$ and $J_{2}$ optimization}

Note that it has been shown in Remark 1 that the relaxation springs in $C 2$ and $C 6$ provide no improvement for ride comfort and Remark 2 also indicates that for better suspension 


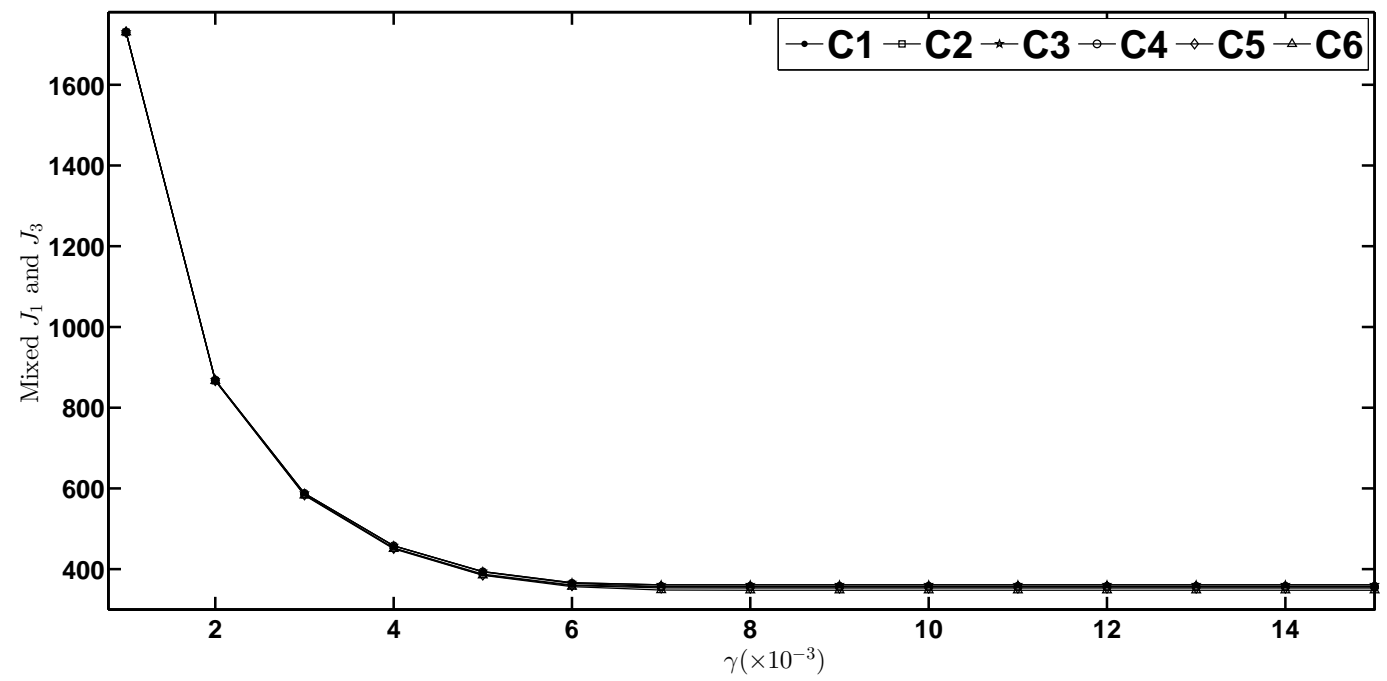

(a)

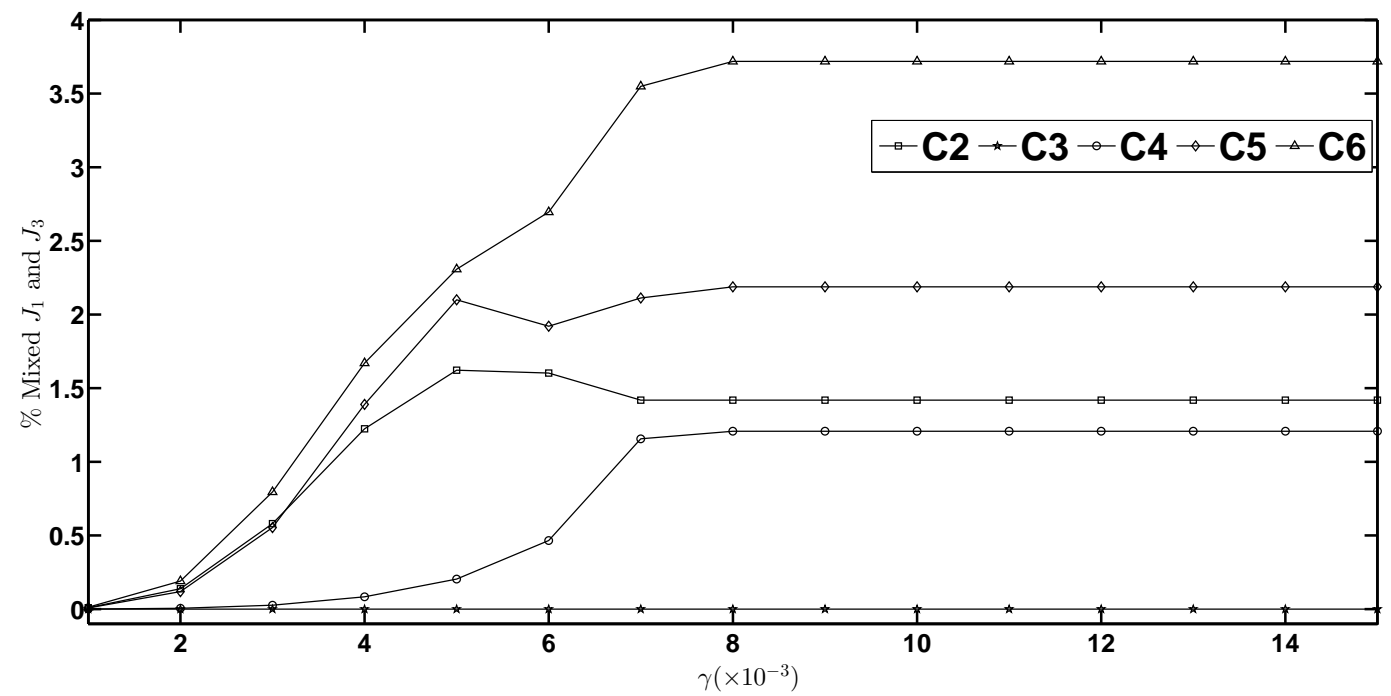

(b)

Figure 7: Mixed $J_{1}$ and $J_{3}$ optimization with equal $J_{2}$ performance requirement when $K=20 \mathrm{kNm}^{-1}$. (a) Mixed performance measure; (b) percentages over $C 1$ of mixed $J_{1}$ and $J_{3}$ performance measure. 


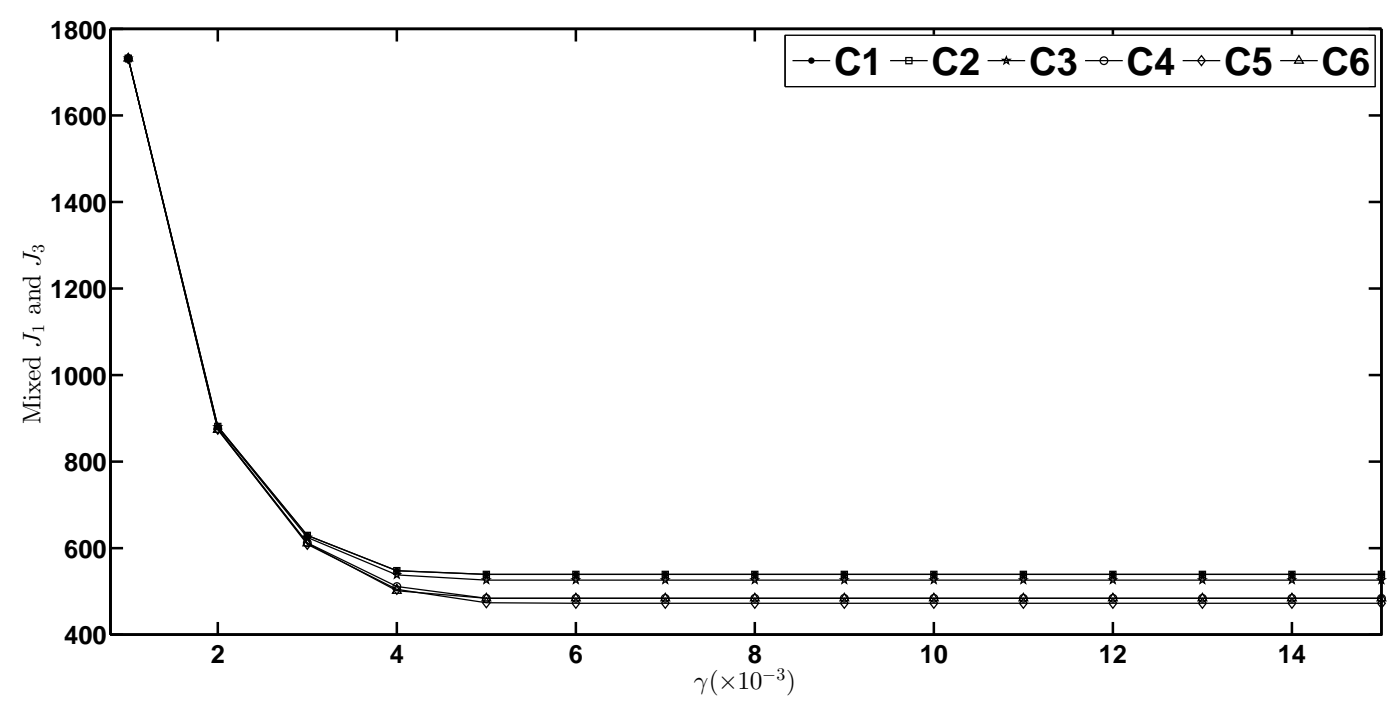

(a)

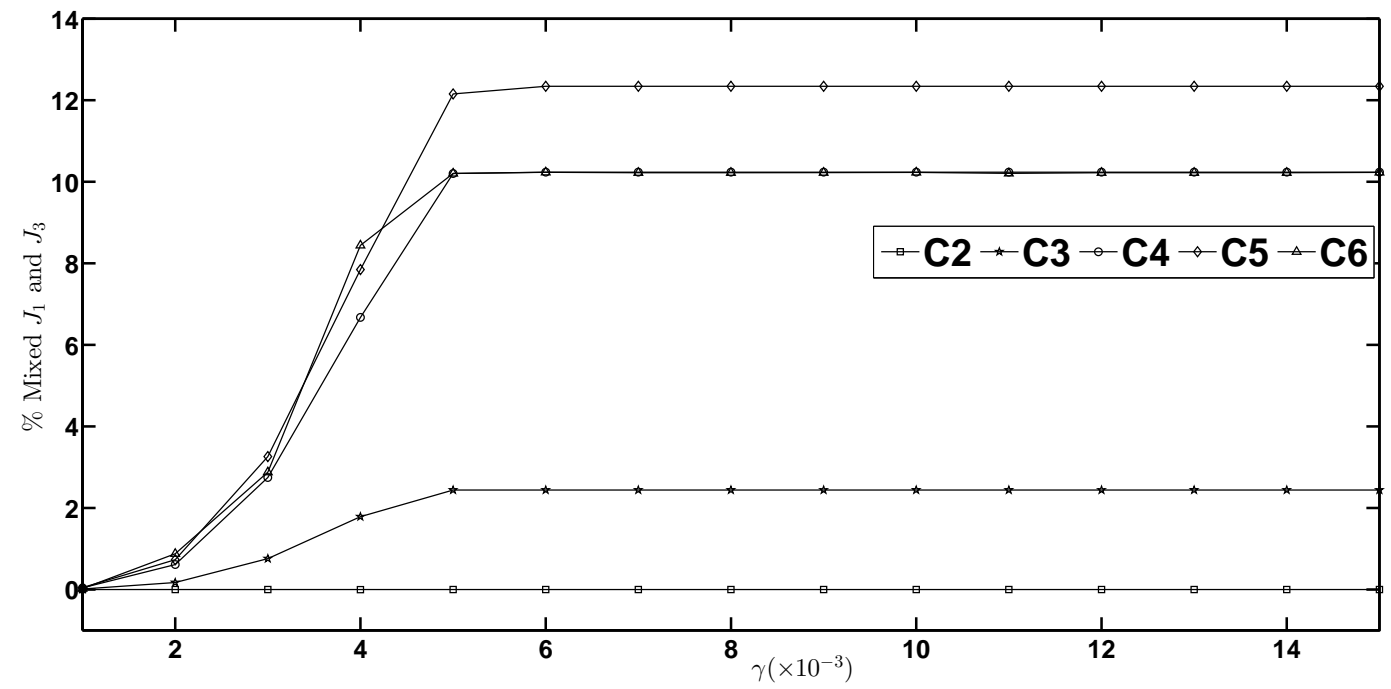

(b)

Figure 8: Mixed $J_{1}$ and $J_{3}$ optimization with equal $J_{2}$ performance requirement when $K=80 \mathrm{kNm}^{-1}$. (a) Mixed performance measure; (b) percentages over $C 1$ of mixed $J_{1}$ and $J_{3}$ performance measure. 


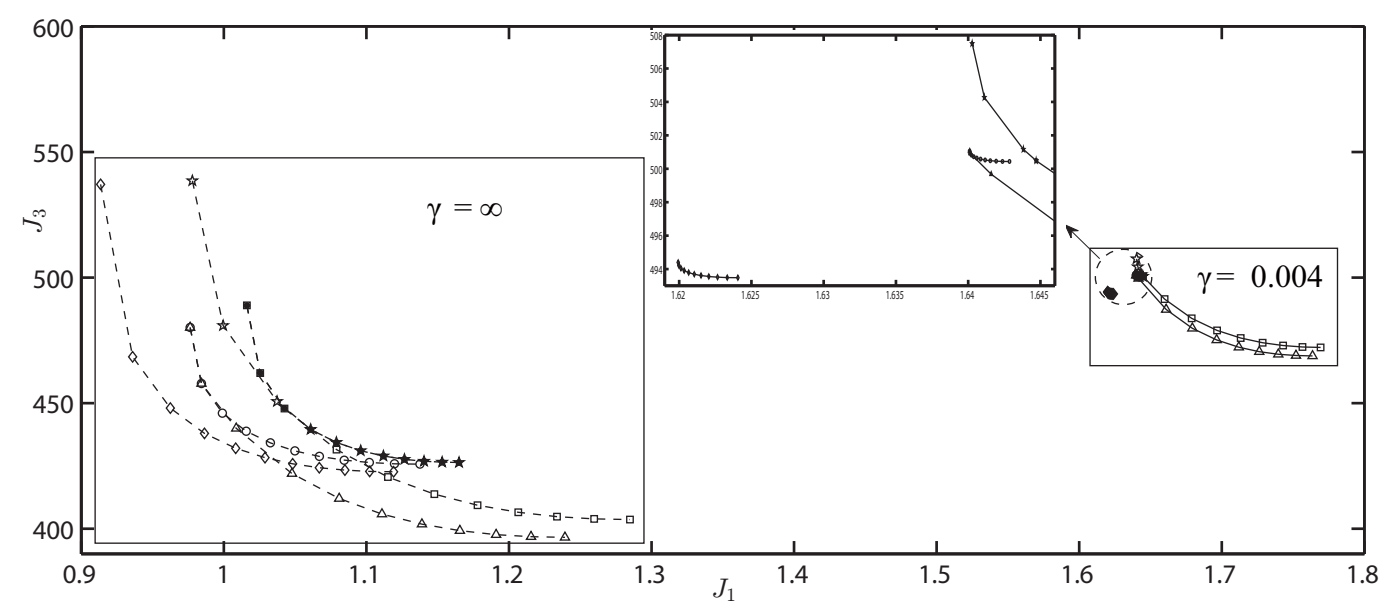

Figure 9: Pareto optimal solutions for $J_{1}$ and $J_{3}$ with and without equal suspension deflection requirement when $K=20 \mathrm{kNm}^{-1}$. Solid and dash lines denote $\gamma=0.004$ and $\gamma=\infty$, respectively. Solid circles denote $C 1$; squares denote $C 2$; Stars denote $C 3$; hollow circles denote $C 4$; diamonds denote $C 5$; triangles denote C6. $\gamma=\infty$ means there is no requirement of equal suspension deflection.

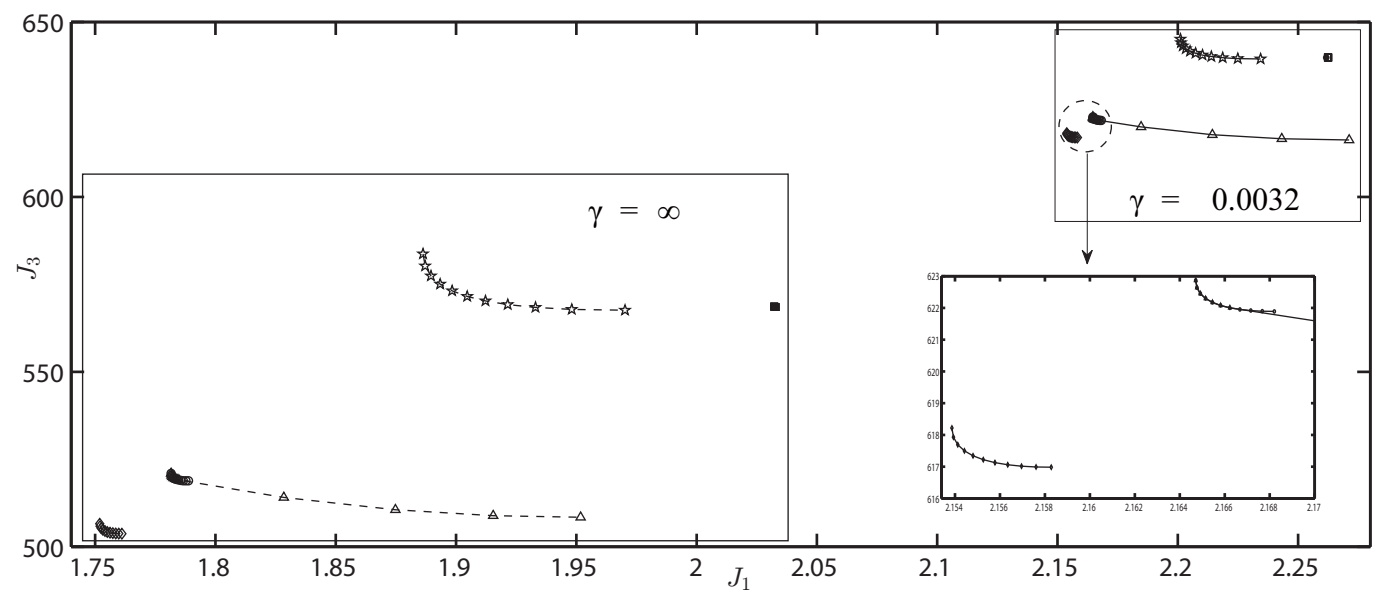

Figure 10: Pareto optimal solutions for $J_{1}$ and $J_{3}$ with and without equal suspension deflection requirement when $K=80 \mathrm{kNm}^{-1}$. Solid and dash lines denote $\gamma=0.0032$ and $\gamma=\infty$, respectively. Solid circles denote $C 1$; squares denote $C 2$; stars denote $C 3$; hollow circles denote $C 4$; diamonds denote $C 5$; triangles denote $C 6$. $\gamma=\infty$ means there is no requirement of equal suspension deflection. 
deflection performance, the relaxation springs should be absolutely stiff. Hence, we deduce that the relaxation springs in $C 2$ and $C 6$ will not improve the mixed performance of $J_{1}$ and $J_{2}$, and only $C 1, C 3, C 4$ and $C 5$ remain to be optimized for mixed $J_{1}$ and $J_{2}$ optimization.

Proposition 1. Let $m_{s}, m_{u}, k_{t}$ be fixed and positive. Consider

$$
H_{C 1: 1,2}=(1-\alpha) H_{C 1 J 1}+\alpha m_{s}^{2} m_{u} H_{C 1 J 2},
$$

where $H_{C 1 J 1}$ and $H_{C 1 J 2}$ are given by Equations (1) and (8).

For any fixed $K$ and $\alpha, H_{C 1 ; 1,2}$ has a unique minimum with

$$
c=\left(\frac{(1-\alpha) d_{2}+\alpha m_{s}^{2} m_{u} e_{1}}{(1-\alpha) d_{1}}\right)^{1 / 2}
$$

Proposition 2. Let $m_{s}, m_{u}, k_{t}$ be fixed and positive. Consider

$$
\begin{aligned}
H_{C 3: 1,2} & =(1-\alpha) H_{C 3 J 1}+\alpha m_{s}^{2} m_{u} H_{C 3 J 2} \\
& =(1-\alpha) d_{1} c+\left((1-\alpha) \frac{g_{3} b^{3}+g_{2} b^{2}+g_{1} b+g_{0}}{f_{1} b+f_{0}}+\alpha m_{s}^{2} m_{u} e_{1}\right) c^{-1}
\end{aligned}
$$

where $H_{C 3 J 1}$ and $H_{C 3 J 2}$ are given by Equations (3) and (10).

For any fixed c, Equation (A.2) has a minimum over $b$ given by $b=0$ or the positive real root of the cubic equation

$$
2 g_{3} f_{1} b^{3}+\left(3 g_{3} f_{0}+g_{2} f_{1}\right) b^{2}+2 g_{2} f_{0} b-g_{0} f_{1}+g_{1} f_{0}=0 .
$$

For any $K \geq 0$ and any $b \geq 0$, the optimal $c$ is given by

$$
c=\left(\frac{(1-\alpha)\left(g_{3} b^{3}+g_{2} b^{2}+g_{1} b+g_{0}\right)+\alpha m_{s}^{2} m_{u} e_{1}\left(f_{1} b+f_{0}\right)}{(1-\alpha) d_{1}\left(f_{1} b+f_{0}\right)}\right)^{1 / 2} .
$$

Proposition 3. Let $m_{s}, m_{u}, k_{t}$ be fixed and positive. Consider

$$
H_{C 4: 1,2}=(1-\alpha) H_{C 4 J 1}+\alpha m_{s}^{2} m_{u} H_{C 4 J 2}
$$

where $H_{C 4 J 1}$ and $H_{C 4 J 2}$ are given by Equations (4) and (11).

For any $K \geq 0$ the optimal $b$ and $c$ to make Equation (A.4) minimal are

$$
\begin{aligned}
b & =-\frac{2\left((1-\alpha) d_{6}+\alpha m_{s}^{2} m_{u} e_{3}\right)}{(1-\alpha) d_{5}} \\
c & =\left(\frac{(1-\alpha) d_{2}+\alpha m_{s}^{2} m_{u} e_{1}}{(1-\alpha) d_{1}+(1-\alpha) d_{5} b^{-1}+\left((1-\alpha) d_{6}+\alpha m_{s}^{2} m_{u} e_{3}\right) b^{-2}}\right)^{1 / 2} .
\end{aligned}
$$

Proposition 4. Let $m_{s}, m_{u}, k_{t}$ be fixed and positive. Consider

$$
H_{C 5: 1,2}=(1-\alpha) H_{C 5 J 1}+\alpha m_{s}^{2} m_{u} H_{C 5 J 2}=c_{f 1} c+c_{r 1} c^{-1},
$$


where $H_{C 5 J 1}$ and $H_{C 5 J 2}$ are given by Equations (5) and (12).

Denote $b_{0}=-\frac{2\left((1-\alpha) d_{6}+\alpha m_{s}^{2} m_{u} e_{3}\right)}{(1-\alpha) d_{5}}$. For any $K \geq 0$ the optimal $k$ and $c$ to make Equation (A.5) minimal are

$$
\begin{aligned}
k & =\frac{(1-\alpha)\left(d_{5}+2 d_{6} b^{-1}\right)+2 \alpha m_{s}^{2} m_{u} e_{3} b^{-1}}{2\left((1-\alpha)\left(d_{7}+d_{8} b^{-1}+d_{9} b^{-2}\right)+\alpha m_{s}^{2} m_{u}\left(e_{4} b^{-2}+e_{5}\right) b^{-1}\right)}, \\
c & =\left(\frac{c_{r 1}}{c_{f 1}}\right)^{1 / 2},
\end{aligned}
$$

where

$$
\begin{aligned}
c_{f 1}= & (1-\alpha) d_{1}+(1-\alpha) d_{5} b^{-1}+\left((1-\alpha) d_{6}+\alpha m_{s}^{2} m_{u} e_{3}\right) b^{-2}, \\
c_{r 1}= & \left((1-\alpha)\left(d_{7}+d_{8} b^{-1}+d_{9} b^{-2}\right)+\alpha m_{s}^{2} m_{u}\left(e_{4} b^{-2}+e_{5} b^{-1}\right)\right) k^{2} \\
& +\left((1-\alpha)\left(-d_{5}-2 d_{6} b^{-1}\right)-2 \alpha m_{s}^{2} m_{u} e_{3} b^{-1}\right) k+(1-\alpha) d_{2}+\alpha m_{s}^{2} m_{u} e_{1},
\end{aligned}
$$

and $b$ is given by the $b_{0}$ or the positive real solution of the function when substituting Equations (A.6) and (A.7) into Equation (A.5) and differentiating with respect to $b$. If the optimal $b$ is larger than $b_{0}$, the optimal $k$ is zero and the network reduces to $C 4$.

\section{Appendix B. Analytical solutions of mixed $J_{2}$ and $J_{3}$ optimization}

Proposition 5. Let $m_{s}, m_{u}, k_{t}$ be fixed and positive. Consider

$$
H_{C 1: 2,3}=(1-\alpha) m_{s}^{4} H_{C 1 J 2}+\alpha H_{C 1 J 3},
$$

where $H_{C 1 J 2}$ and $H_{C 1 J 3}$ are given by Equations (8) and (15).

For any fixed $K$ and $\alpha, H_{C 1 ; 2,3}$ has a unique minimum given by $c=\left(\frac{(1-\alpha) m_{s}^{4} e_{1}+\alpha a_{2}}{\alpha a_{1}}\right)^{1 / 2}$.

Proposition 6. Let $m_{s}, m_{u}, k_{t}$ be fixed and positive. Consider

$$
H_{C 2: 2,3}=(1-\alpha) m_{s}^{4} H_{C 2 J 2}+\alpha H_{C 2 J 3}=c_{f 2} c+c_{r 2} c^{-1},
$$

where $H_{C 2 J 2}$ and $H_{C 2 J 3}$ are given by Equations (9) and (16) and

$$
c_{f 2}=\alpha a_{1}+\alpha a_{3} k^{-1}+\left((1-\alpha) m_{s}^{4} e_{2}+\alpha a_{4}\right) k^{-2}, \quad c_{r 2}=(1-\alpha) m_{s}^{4} e_{1}+\alpha a_{2} .
$$

Denote $K_{1}=\frac{m_{s}\left(m_{s}+2 m_{u}\right) k_{t}}{2\left(m_{s}+m_{u}\right)^{2}}$. The optimal value of $k$ and $c$ where $H_{C 2 ; 2,3}$ achieves its minimum are given by

$$
k^{-1}=\left\{\begin{array}{ll}
-\frac{\alpha a_{3}}{2\left((1-\alpha) m_{s}^{4} e_{2}+\alpha a_{4}\right)}, & K<K_{1} \\
0, & K \geq K_{1}
\end{array} \quad \text { and } c=\left(\frac{c_{r 2}}{c_{f 2}}\right)^{1 / 2} .\right.
$$

Proposition 7. Let $m_{s}, m_{u}, k_{t}$ be fixed and positive. Consider

$$
\begin{aligned}
H_{C 3: 2,3} & =(1-\alpha) m_{s}^{4} H_{C 3 J 2}+\alpha H_{C 3 J 3}, \\
& =\alpha a_{1} c+\left((1-\alpha) m_{s}^{4} e_{1}+\alpha\left(a_{2}-a_{3} b+a_{5} b^{2}\right)\right) c^{-1},
\end{aligned}
$$

where $H_{C 3 J 2}$ and $H_{C 3 J 3}$ are given by Equations (10) and (17).

The optimal value of $b$ and $c$ where $H_{C 3: 2,3}$ achieves its minimum is given by

$$
b=\left\{\begin{array}{ll}
\frac{a_{3}}{2 a_{5}}, & K<K_{1} \\
0, & K \geq K_{1}
\end{array} \quad \text { and } c=\left(\frac{(1-\alpha) m_{s}^{4} e_{1}+\alpha\left(a_{2}-a_{3} b+a_{5} b^{2}\right)}{\alpha a_{1}}\right)^{1 / 2} .\right.
$$


Proposition 8. Let $m_{s}, m_{u}, k_{t}$ be fixed and positive. Consider

$$
H_{C 4: 2,3}=(1-\alpha) m_{s}^{4} H_{C 4 J 2}+\alpha H_{C 4 J 3}=c_{f 3} c+c_{r 3} c^{-1}
$$

where $H_{C 4 J 2}$ and $H_{C 4 J 3}$ are given by Equations (11) and (18) and

$$
c_{f 3}=\alpha a_{1}+\alpha a_{6} b^{-1}+\left((1-\alpha) m_{s}^{4} e_{3}+\alpha a_{7}\right) b^{-2}, \text { and } c_{r 3}=(1-\alpha) m_{s}^{4} e_{1}+\alpha a_{2} .
$$

Denote $K_{2}=\frac{m_{s} m_{u} k_{t}}{\left(m_{s}+m_{u}\right)^{2}}$. The optimal value of $b$ and $c$ where $H_{C 4 ; 2,3}$ achieves its minimum is given by

$$
b^{-1}=\left\{\begin{array}{ll}
-\frac{\alpha a_{6}}{2\left((1-\alpha) m_{s}^{4} e_{3}+\alpha a_{7}\right)}, & K<K_{2} \\
0, & K \geq K_{2}
\end{array} \quad \text { and } c=\left(\frac{c_{r 3}}{c_{f 3}}\right)^{1 / 2} .\right.
$$

Proposition 9. Let $m_{s}, m_{u}, k_{t}$ be fixed and positive. Consider

$$
H_{C 5: 2,3}=(1-\alpha) m_{s}^{4} H_{C 5 J 2}+\alpha H_{C 5 J 3}=c_{f 4} c+c_{r 4} c^{-1},
$$

where $H_{C 5 J 2}$ and $H_{C 5 J 3}$ are given by Equations (12) and (19) and

$$
\begin{aligned}
c_{f 4} & =\alpha a_{1}+\alpha a_{6} b^{-1}+\left((1-\alpha) m_{s}^{4} e_{3}+\alpha a_{7}\right) b^{-2}, \quad c_{r 4}=t_{2} k^{2}+t_{1} k+t_{0}, \\
t_{2} & =\alpha a_{8}+\left((1-\alpha) m_{s}^{4} e_{5}+\alpha a_{9}\right) b^{-1}+\left((1-\alpha) m_{s}^{4} e_{4}+\alpha a_{10}\right) b^{-2}, \\
t_{1} & =-\left(\alpha a_{6}+\left(2(1-\alpha) m_{s}^{4} e_{3}+2 \alpha a_{7}\right) b^{-1}\right), \quad t_{0}=(1-\alpha) m_{s}^{4} e_{1}+\alpha a_{2} .
\end{aligned}
$$

Denote $b_{1}=\frac{-2\left((1-\alpha) m_{s}^{4} e_{3}+\alpha a_{7}\right)}{\alpha a_{6}}$. For any $K$ and $\alpha$, the optimal value of $k, b$, and $c$ where $H_{C 5: 2,3}$ achieves its minimum are given by

$$
k=-\frac{t_{1}}{2 t_{2}}, \quad c=\left(\frac{c_{r 4}}{c_{f 4}}\right)^{1 / 2} .
$$

Let $\mathcal{Q}$ be the set of positive real solutions b of the equation after substituting Equation (B.6) into Equation (B.5) and differentiating with respect to $b$. The optimal value of $b$ is equal to $b_{1}$ or $\mathcal{Q} \bigcap\left(0, b_{1}\right)$. If $b=\infty, C 5$ reduces to $C 1$, and if $k=0, C 5$ reduces to $C 4$.

Proposition 10. Let $m_{s}, m_{u}, k_{t}$ be fixed and positive. Consider

$$
H_{C 6: 2,3}=(1-\alpha) m_{s}^{4} H_{C 6 J 2}+\alpha H_{C 6 J 3}=c_{f 5} c+c_{r 5} c^{-1},
$$

where $H_{C 6 J 2}$ and $H_{C 6 J 3}$ are given by Equations (13) and (20).

For any fixed $K$ and $\alpha, H_{C 6: 2,3}$ has a unique minimum over $b, c$ and $k$ at

$$
k=-\frac{2\left((1-\alpha) m_{s}^{4} e_{2}+\alpha a_{4}\right)}{-2(1-\alpha) m_{s}^{4} e_{1} b^{-1}+\alpha\left(a_{3}-2 a_{2} b^{-1}\right)}, \quad b^{-1}=\frac{b_{n u m}^{-1}}{b_{d e n}^{-1}} \text { and } c=\left(\frac{c_{r 5}}{c_{f 5}}\right)^{1 / 2},
$$

with $b, k \geq 0$ and

$$
\begin{aligned}
b_{\text {num }}^{-1} & =\alpha\left(a_{6} e_{2} m_{s}^{4}-a_{6} e_{2} m_{s}^{4} \alpha+a_{6} a_{4} \alpha+a_{3} e_{1} m_{s}^{4}-a_{3} e_{1} m_{s}^{4} \alpha+a_{3} a_{2} \alpha\right), \\
b_{d e n}^{-1} & =\left(-2 m_{s}^{8} e_{3} e_{2}-2 a_{7} a_{4}+2 m_{s}^{4} e_{3} a_{4}+2 a_{7} m_{s}^{4} e_{2}+2 m_{s}^{8} e_{1}^{2}-4 m_{s}^{4} e_{1} a_{2}+2 a_{2}^{2}\right) \alpha^{2} \\
+ & \left(4 m_{s}^{8} e_{3} e_{2}-2 m_{s}^{4} e_{3} a_{4}-2 a_{7} m_{s}^{4} e_{2}+4 m_{s}^{4} e_{1} a_{2}-4 m_{s}^{8} e_{1}^{2}\right) \alpha-2 m_{s}^{8} e_{3} e_{2}+2 m_{s}^{8} e_{1}^{2} .
\end{aligned}
$$

If $b=\infty$ or $k=\infty$ at any global minimum, the $C 6$ configuration reduces to the case of $C 2$ or $C 4$, respectively. 


\section{References}

[1] M.C. Smith, G.W. Walker, Performance limitations and constraints for active and passive suspensions: A mechanical multi-pot approach, Vehicle System Dynamics 33 (2000) $137-168$.

[2] M.C. Smith, Synthesis of mechanical networks: The inerter, IEEE Transactions on Automatic Control 47 (10) (2002) 1648-1662.

[3] H. Du, N. Zhang, F. Naghdy, Robust control of vehicle electrorheological suspension subject to measurement noises, Vehicle System Dynamics 49 (2011) 257-275.

[4] H. Du, K.Y. Sze, J. Lam, Semi-active $H_{\infty}$ control of vehicle suspension with magnetorheological dampers, Journal of Sound and Vibration 283 (5) (2005) 981-996.

[5] H. Du, N. Zhang, $\mathrm{H}_{\infty}$ control of active vehicle suspensions with actuator time delay, Journal of Sound and Vibration 301 (1) (2007) 236-252.

[6] H. Du, N. Zhang, J. Lam, Parameter-dependent input-delayed control of uncertain vehicle suspensions, Journal of Sound and Vibration 317 (3) (2008) 537-556.

[7] M.Z.Q. Chen, C. Papageorgiou, F. Scheibe, F.C. Wang, M.C. Smith, The missing mechanical circuit element, IEEE Circuits and System Magazine 9 (1) (2009) 10-26.

[8] M.C. Smith, F.C. Wang, Performance benefits in passive vehicle suspensions employing inerters, Vehicle System Dynamics 42 (4) (2004) 235-257.

[9] F. Scheibe, M.C. Smith, Analytical solutions for optimal ride comfort and tyre grip for passive vehicle suspensions, Vehicle System Dynamics 47 (10) (2009) 1229-1252.

[10] F.C. Wang, W.J. Sue, The impact of inerter nonlinearities on vehicle suspension control, Vehicle System Dynamics 46 (7) (2008) 575-595.

[11] C. Papageorgiou, M.C. Smith, Positive real synthesis using matrix inequalities for mechanical networks: application to vehicle suspension, IEEE Transactions on Control Systems Technology 14 (3) (2006) 423-435.

[12] M.Z.Q. Chen, Y. Hu, B. Du, Suspension performance with one damper and one inerter, Proceedings of the 24th Chinese Control and Decision Conference (CCDC), Tainyuan, China, 2012, pp. 3551-3556.

[13] F.C. Wang, H.A. Chan, Mechatronic suspension design and its applications to vehicle suspension control, Proceedings of the 47th IEEE Conference on Decision and Control, Cancun, Mexico, 2008, pp. 3769-3774.

[14] F.C. Wang, H.A. Chan, Vehicle suspensions with a mechatronic network strut, Vehicle System Dynamics 49 (5) (2011) 811-830.

[15] S. Evangelou, D.J.N. Limebeer, R.S. Sharp, M.C. Smith, Control of motorcycle steering instabilities, IEEE Control Systems Magazine, 26 (5) (2006) 78-88. 
[16] S. Evangelou, D.J.N. Limebeer, R.S. Sharp, M.C. Smith, Mechanical steering compensators for high-performance motorcycles, Journal of Applied Mechanics 74 (2) (2007) $332-336$.

[17] F.C. Wang, M.K. Liao, B.H. Liao, W.J. Sue, H.A. Chan, The performance improvements of train suspension systems with mechanical networks employing inerters, Vehicle System Dynamics 47 (7) (2009) 805-830.

[18] F.C. Wang, M.K. Liao, The lateral stability of train suspension systems employing inerters, Vehicle System Dynamics 48 (5) (2009) 619-643.

[19] F.C. Wang, M.R. Hsieh, H.J. Chen, Stability and performance analysis of a full-train system with inerters, Vehicle System Dynamics 50 (4) (2011) 545-571.

[20] J.Z. Jiang, A.Z. Matamoros-Sanchez, R.M. Goodall, M.C. Smith, Passive suspensions incorporating inerters for railway vehicles, Vehicle System Dynamics 50 (sup1) (2012) 263-276.

[21] F.C. Wang, C.W. Chen, M.K. Liao, M.F. Hong, Performance analyses of building suspension control with inerters, Proceedings of the 46th IEEE Conference on Decision and Control, New Orleans, LA, USA, 2007, pp. 3786-3791.

[22] F.C. Wang, M.F. Hong, C.W. Chen, Building suspensions with inerters, Proceedings of the IMechE, Part C: Journal of Mechanical Engineering Science 224 (8) (2010) 16051616.

[23] M.Z.Q. Chen, Passive Network Synthesis of Restricted Complexity, PhD Thesis, Cambridge Univ. Eng. Dept., U.K., 2007.

[24] M.Z.Q. Chen, M.C. Smith, Restricted complexity network realizations for passive mechanical control, IEEE Transactions on Automatic Control 54 (10) (2009) 2290-2301.

[25] M.Z.Q. Chen, K. Wang, Z. Shu, C. Li, Realizations of a special class of admittances with strictly lower complexity than canonical forms, IEEE Transactions on Circuits and Systems-I: Regular Papers 60 (9) (2013) 2465-2473.

[26] M.Z.Q. Chen, K. Wang, Y. Zou, and J. Lam, "Realization of a special class of admittances with one damper and one inerter for mechanical control," IEEE Transations on Automatic Control, 58 (7) (2013) 1841-1846.

[27] J.Z. Jiang, M.C. Smith, Regular positive-real functions and five-element network synthesis for electrical, IEEE Transactions on Automatic Control 56 (6) (2011) 1275-1290.

[28] R.S. Sharp, D.A. Crolla, Road vehicle suspension system design-A review, Vehicle System Dynamics 16 (3) (1987) 167-192.

[29] R.S. Sharp, S.A. Hassan, The fundamental of passive automotive suspension system design, Society of Environmental Engineers Conference on Dynamics in Automotive Engineering, 1984, pp. 104-115. 
[30] R.S. Sharp, S.A. Hassan, The relative performance capabiblities of passive, active and semi-active car suspension systems, Proceedings of Insttitution of Mechanical Engineers 200 (3) (1986) 219-228.

[31] R.S. Sharp, S.A. Hassan, An evaluation of passive automotive suspension systems with variable stiffness and damping parameters, Vehicle System Dynamics 15 (6) (1986) 335350 .

[32] G.W. Walker, Constraints upon the Achievable Performance of Vehicle Suspension Systems, PhD Thesis, Cambridge Univ. Eng. Dept., U.K., 1997. 